\title{
Agreement of co-operation between Dharma Chitra Guthi and Nepal Medical Association
}

1. Dharma Chitra Guthi, a charitable institution or trust, desirous of promoting the advancement of medical knowledge for the benefit of the ailling and diseased, hereby expresses its willingnesss to sign an Agreement of Co-operation with the Nepal Medical Association, which is devoted to the cause of Medicine and of its menber who a:e professionals in the field of Medicine.

2. The Nepal Medical Association shall from a MEDICAL TRUST to be called THE NEPAL. MEDICAL ASSOCIATION MEDICAL TRUST.

3. The NEPAL MEDICAL ASSOCIATION MEDICAL TRUST shall be operated by a Governing Board consisting 5 Trustees: 2 Trustees shall be nominated by the Executive Committee of The Nepal Medical Association; 2 Trustees shall be nominated by the Governing Body of Dharma Chitra Gushi: The fifth Trustee who shall bo Chairman of the NMA Medical Trust shall be nominated jointly by the Nepal Medical Association and Dharma Chitra Guthi. The Chairman shall be an independent person, not belonging to either the Nepal Medical Association or Dharma Chitra Guthi, who need not necessarily be a Nepali citizen but shall be mutually acceptable to both parties.

4. Mrs. Chitra Kumari Pahari, on behalp of Dharma Chitra Guthi, shall pay into the account of the NMA Medical Trust a sum of Rupces Three Hundred Thousand only which shall be placed in an interest earning deposit in a bank or any other institution or organisation which is mutually agreed upon as secure.

5. The interest accuring from this deposit shell be used by the NMA Medical Trust in presenting various AWARDS to Medical Doctor or Paramedical working in the fields of Medicine and Health, who in the opinion of The Trustees of the NMA Medical Trust. have made significant contribution in the field of Modicine and Health. The prizes which shall be awarded every two years during the Inaugural Ceremony of All Neral Medical Conference, will consist of: 
(a) AMAR PAHARI MEDICAL LITERARY A.WARD - This awad shall consist of cash prize of Fs. 20,000/-and a certificate which will be given to the best medical book writien by a Nepali Doctor or a non-Docior who is involved in the ficld of modicine and health.

(b) DHARIMA PAHARI HEALTH SERVICE AWARD - This shall consist of cash prizes of Rs. 25,000/-and a certificate, which will be given to distingushed Loctor of Non-Doctor who in the opinion of the Trustees has mate outstanding contribution for the cause of medicine and health in Nepol.

(c) CHITRA PAHARI SCIENTIFIC MEDICAL AWARD. This shalt consist of cash prizes of Rs. 5,000/-and 3,000/-which will be given to the authors of the First and Second best articles in the Medical Journats published in Nepal.

6. Any oxcess balance available after the payment of the award and all incidental expenses shall be utilized for other medical purpoges, as par the decision of the Trustees.

Signed by Mrs. Chitra Kumari Pahari on behalf of Dherma Chitra Guthi.

Dr. S. K. Pahari, witness

Dated:- 2/12/2041
Signed by Dr. B. B. Vaidya President. on behalf of Nepal Medical Association

Dr. S. K. Bhatracharya. President Elect, Witness

Dated:- 2/12/2041 


\section{नेपाल चिकित्सक संघ}

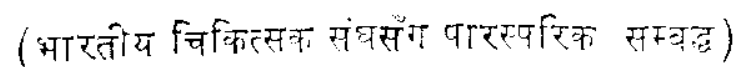
केश्द्रीय कार्यासच, सिद्ध सदन

हलाक मंजुसा १कहो, काठमाण्डू

81. चेतराज प्त्त

27. गोविन्द्ध घल्लभ भट्ट

37. पूरन बज्ताचार्य

हा. शंलेश जपाध्याय

प. सं. २०४र ने. मे. ए रारशट

zा. विन्देश्वर महतं

31. विमल छकाल

उा. पत्वन क्यार गर्ग

हा. जे. पो. वराल

विवय:- नेपाल चिकित्सक संघ, लुक्यनी अच्चल शासा सोल्न सदीकृत

महाशयहस,

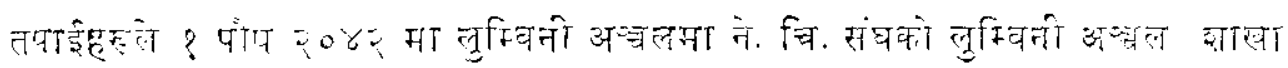

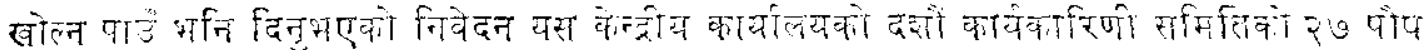

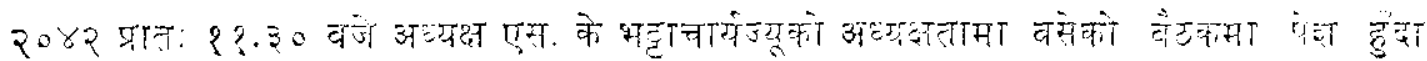

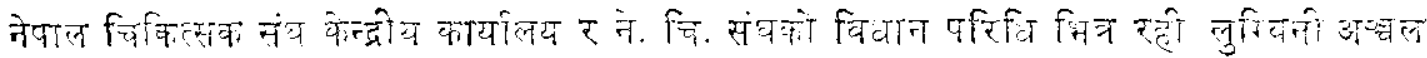

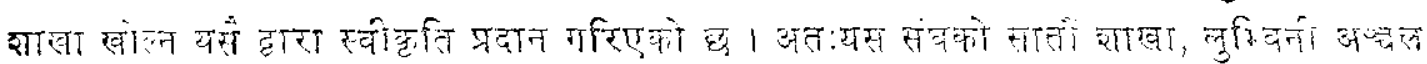

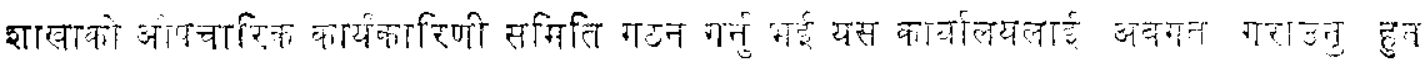
अनुरोध गद्दें।

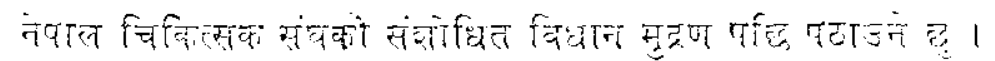

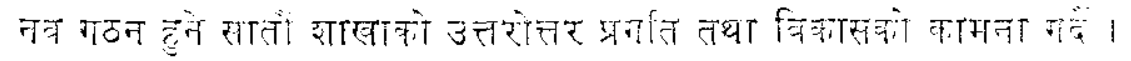

बोधार्थ:-

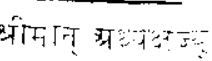

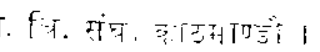

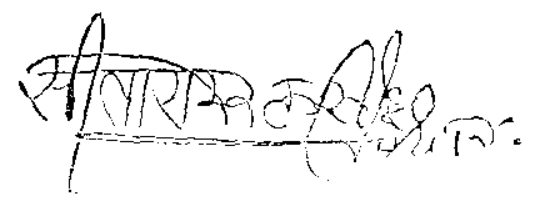

डा. सौसारम नि. एल प्रवान

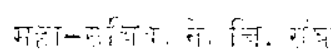



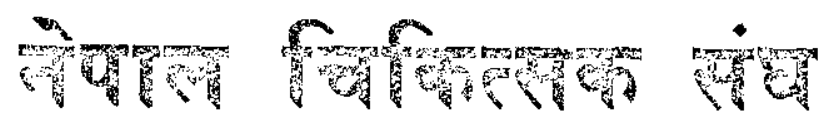

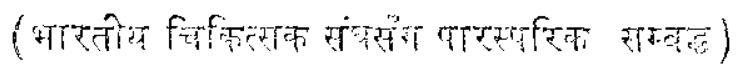

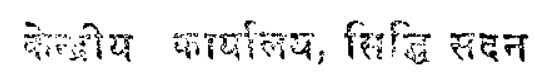

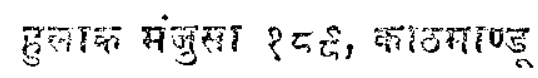

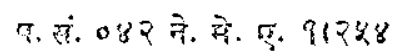

किति र००रा१०एर्षाप मा

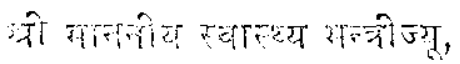

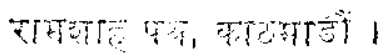

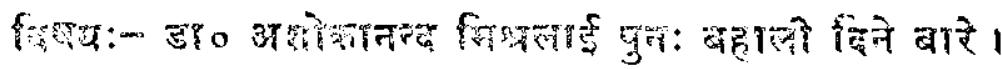

महंदय,

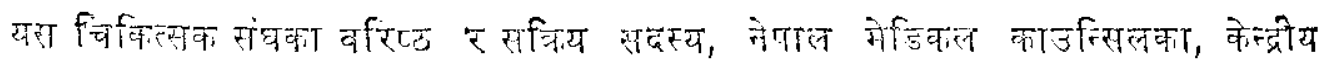

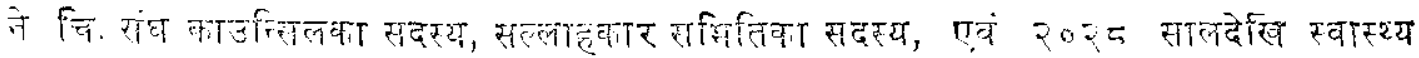

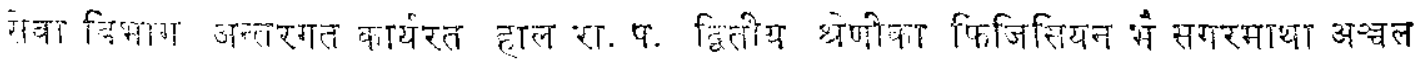

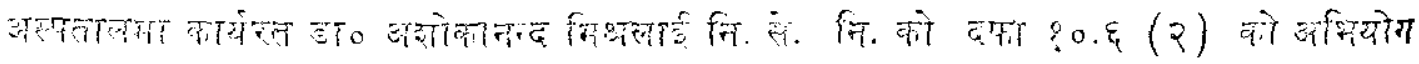

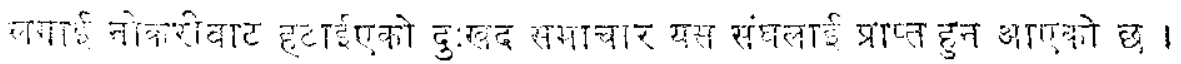

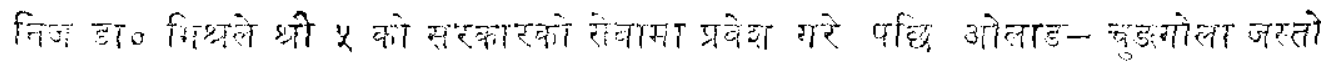

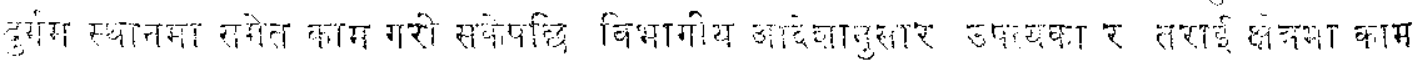

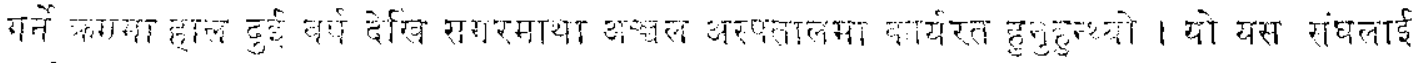

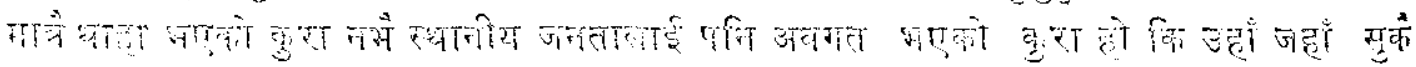

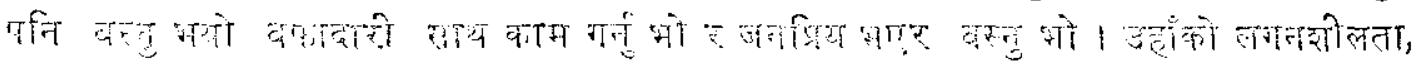

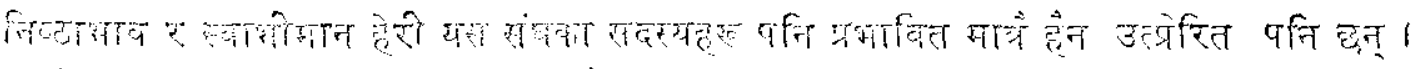

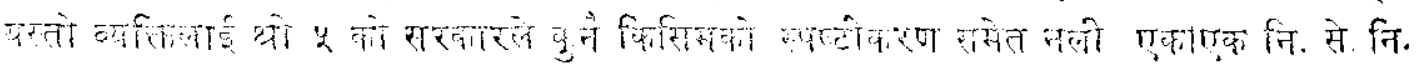




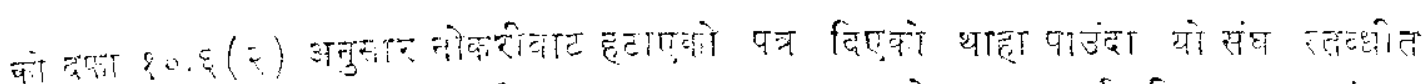

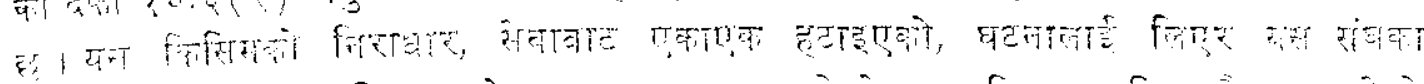

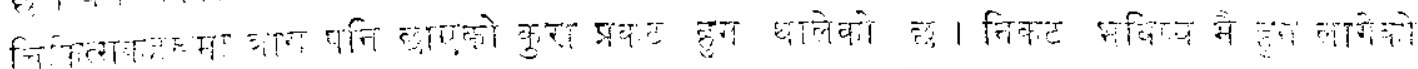

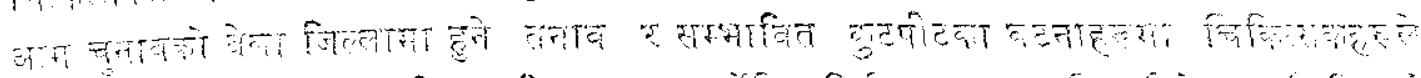

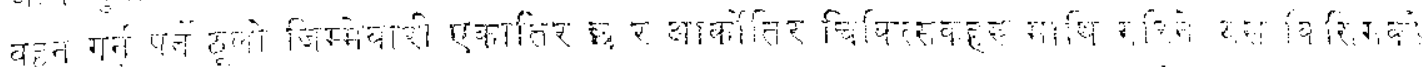

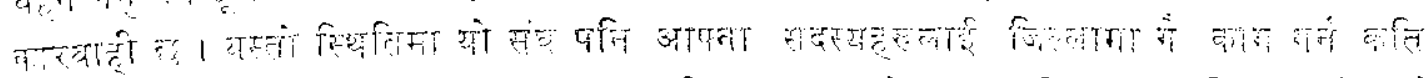

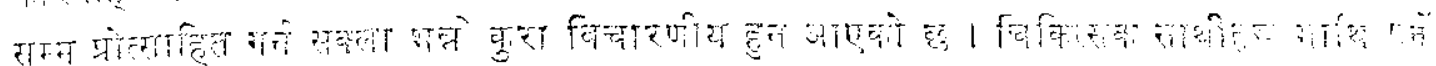

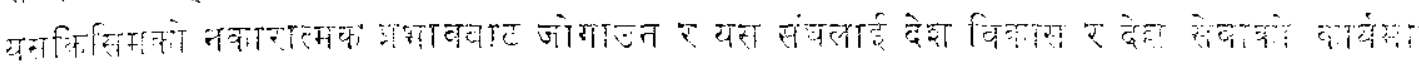

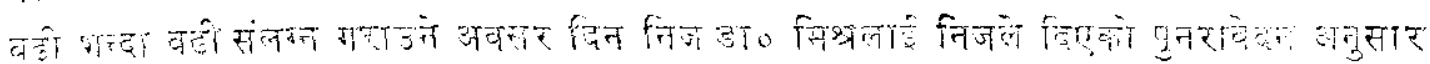

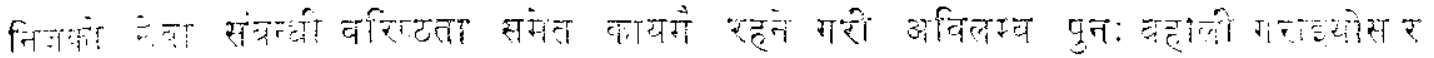

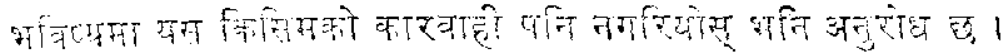

भवर्वर्ब

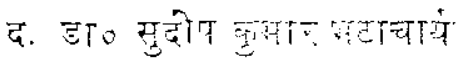
स्रंज्वा

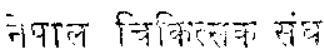

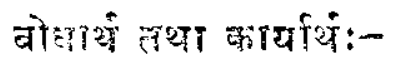

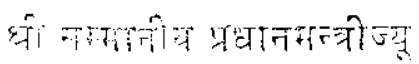

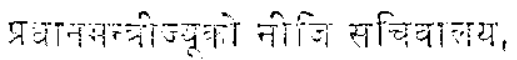

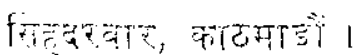

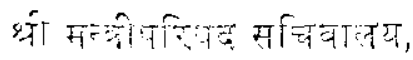

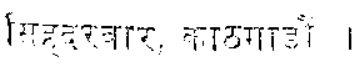

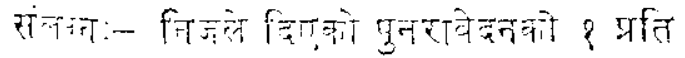




\title{
नेषल चिकित्सक संध
}

(गरितोय चिकित्सक संघसंग गरस्परिक सम्बद्ध)

\author{
केन्द्रीय कार्यालय सिद्धि सदन
}

हुलाक मंजुसा ?दर्द, काठमाण्डू

प. सं. ०४ २ ने. चि. स. २१३०३

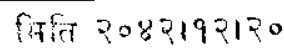

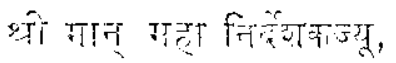

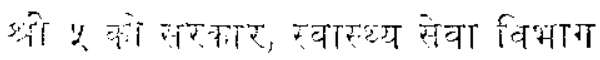

ऐलि कागमाहैं।

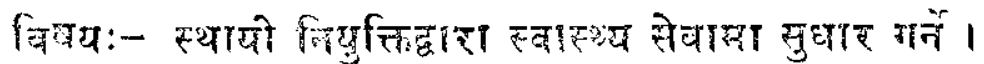

गहिंद्य,

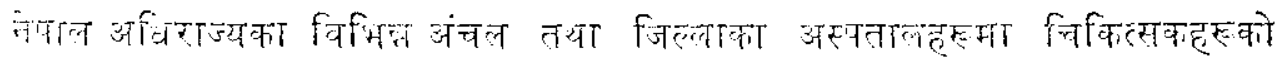

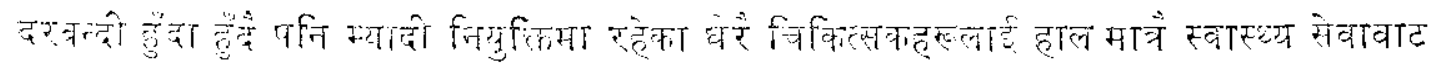

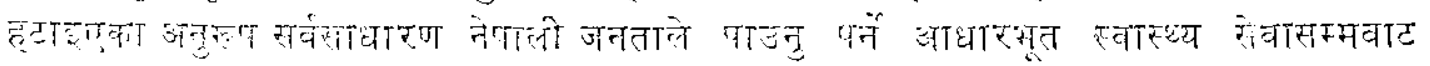

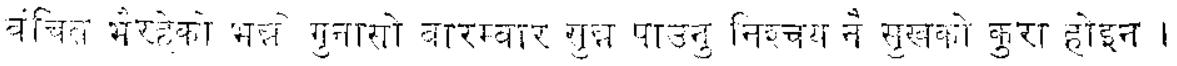

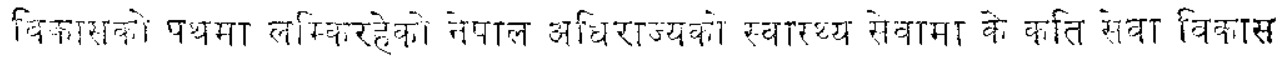

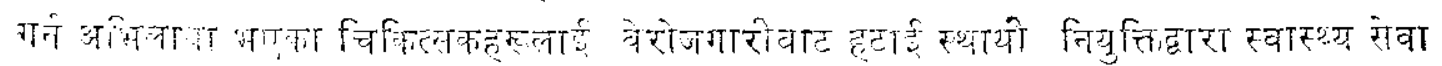

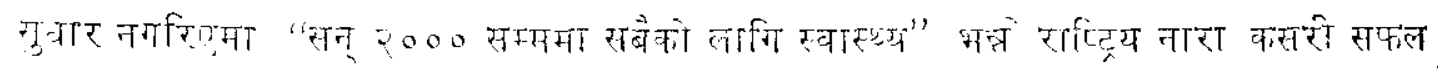

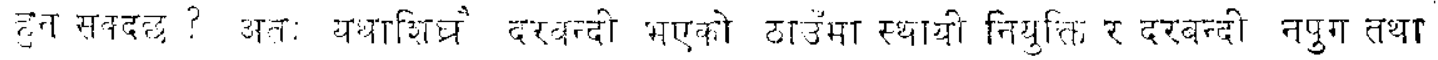

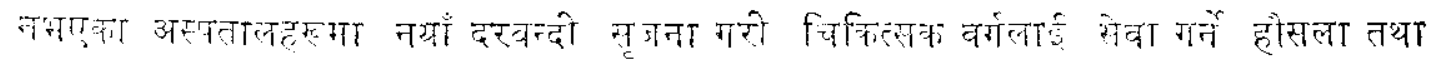

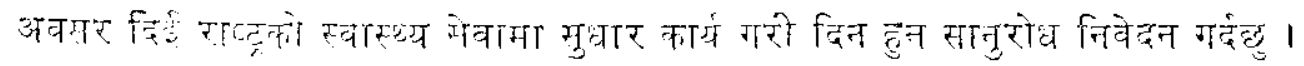

बोधार्थ:-

श्रीमान् अध्यक्ष ज्यु,

नेषाल नितिंत्सक गंघ

प्रदर्रानी मार्ग, काटमाडों।

\section{भवर्वाग}

ज० सीत्राराम प्रचान

गद्या-सिचिन

नेषगल चिनिसक संध 


\section{Suspended Members: Please Renew Your Membership}

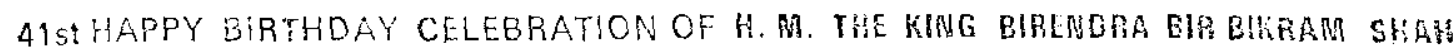
DEV: NMA celebrated the 4 Ist Happy Birthday of $\mathrm{H}$, W. The King Birendra Bir Eikram Shah Dev at the NMA lawn on 13 thi Poush amidst distinguished dignitaries and many memiders of this Association. The President Dr. S. K. Bhattacharya proposed a toast to the Health and Happiness of His Majesty the King, to the peace, progress and better health services of Nation.

ICSiNMA Setellite Netro Convention: Internationa! Coilege of Surgeons, Nepal Seciicn and NMA organised a Satellite Neuro Convention at Hotel Blue Ster on 2 Ist December 1985 where Dr. D. N. Gongal from Nepai, Dr. K. V. Mathai, Dr. H.S. Subramaniam, Dr. K. Ganapaty and Dr. M. Sarribasivam from different states of India contributed talks on advances of Neurology.

Talk on Recent Aduances in Iepatabilliary Surgery: On NMA's organisation Piof. L. H. Elumgart, Hepatobilliary Surgcon, Hemmersmith Hospital, Loncon gevea short talk on Recent Advances in Hepatobillialy Surgery in tl:e Eir Hospitai auditorium on Sth Falgun 2042.

9th Honcrary Nifia Menher: Mr. Ryutaro Hashimoto, Member of House of Representatives, Japanese Diet, ex-Minister for Healuh, Japan was conferred Honorary Membership on 26 th February 1986 in a special celemony heid at NMA auditorium.

Eongratulations to: Dr. Y.M. Prodhan, the leader of the Eye Camp at Dang Deukuri What a Team from AOCA, Jepan headed by Er. liaru Kurczumi on successiully completing $12 y$ cataract operations botween 15 th Poush and 25 th Polich this year.

\begin{tabular}{|c|c|c|c|}
\hline Now rongurs: & s. & Mem No. & Name of tha flamtis \\
\hline & 1. & 919: L-17! & Dr. Leonardo Juan Vigna \\
\hline & 2. & 920 & Dr. Gajendra Prasad \\
\hline & 3. & 921 & Dr. Gopal Prasad Khanal \\
\hline & 4. & 022 & Dr. Mahende Keshar Chtote \\
\hline
\end{tabular}




\begin{tabular}{|c|c|c|}
\hline 5. & 923 & Dr. Raj Sokhar Jha \\
\hline 6. & 924 & Dr. Madhur Dev Bhattarai \\
\hline 7. & 925 & Dr. Gopal Prasad Sah \\
\hline 8. & 926 & Dr. Chandra Karki \\
\hline 9. & $927 / L-127$ & Dr. Tapeshwar Lal Karna \\
\hline 10. & 928 & Dr. Arjun Raj Pant \\
\hline 11. & $920 / 1.173$ & Dr. Ram Lakhan Shoh \\
\hline 12 & $9>0$ & Dr. Chadra Kane Sharma \\
\hline 13 & 931 & Di. Sira Pant Choudary \\
\hline 14 & $33 / L-174$ & Dr. Antrew Samed Fober \\
\hline 15 & 933 & Dr. Ramji Chaudary \\
\hline 13 & 934 & Dr. Binod Lel Defromaya \\
\hline 7 & $679 \mathrm{~L}-169$ & Cr. Bol krishan Snbodi \\
\hline 18. & $366:-170$ & Dr. Dirga Gigh Gan \\
\hline
\end{tabular}

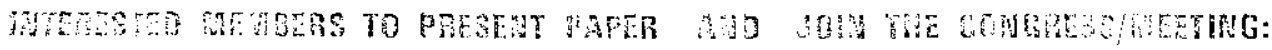

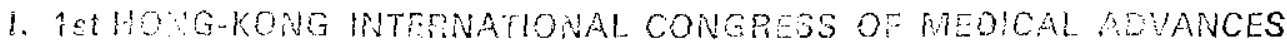
21-2. September 1906

Crstater

The Hora Kong Medical Association

Suke of Wircisor Building

5 th Floor. 15 Hennossy Road

P. O. BOXNO. 1957, HONG KONG.

11. 6th BIENHAAL GENERAL SCIENTIFIC MEETING, BANCKOK, THAILAND

Fobruary 8-13, 1987

\section{Contect To}

Tile Secretariat 6th BGSM, ASSEA

P. $0.80 \times 57$

BKK 10300, Thailand.

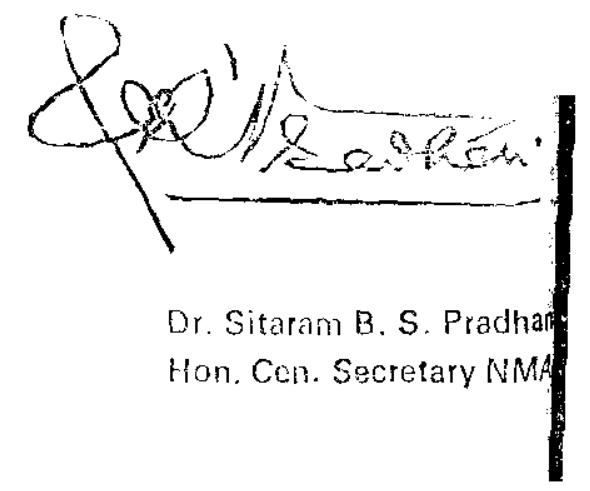

$-66-$ 
Vay Peace Prevail on the Departed Souls:

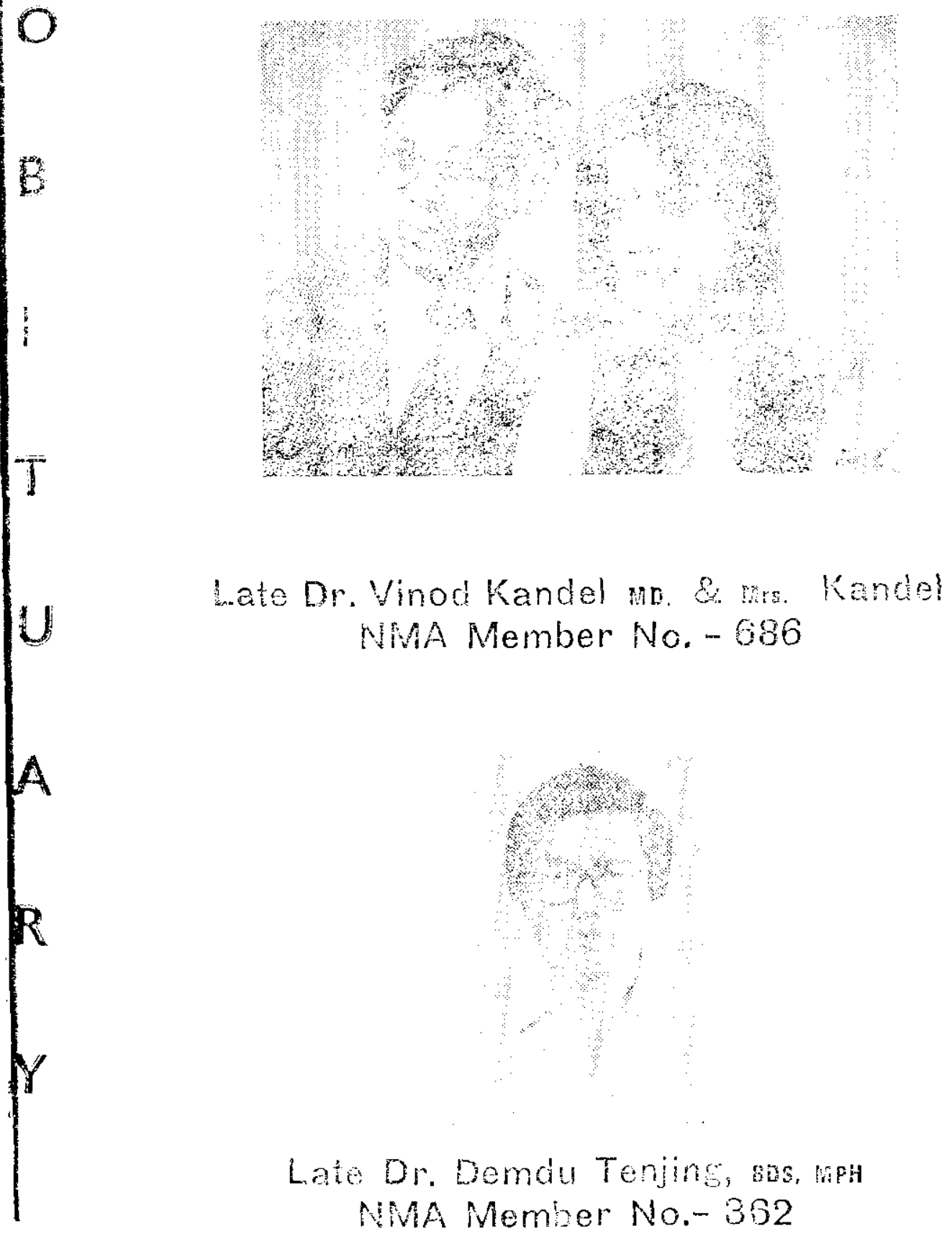

